Article

\title{
Microwave Assisted Synthesis, Antifungal Activity and DFT Theoretical Study of Some Novel 1,2,4-Triazole Derivatives Containing the 1,2,3-Thiadiazole Moiety
}

\author{
Na-Bo Sun ${ }^{1}$, Jian-Qun Fu ${ }^{1}$, Jian-Quan Weng ${ }^{2}$, Jian-Zhong Jin ${ }^{1}$, Cheng-Xia Tan ${ }^{2}$ and \\ Xing-Hai Liu ${ }^{2, *}$
}

1 College of Biology and Environmental Engineering, Zhejiang Shuren University, Hangzhou 310015, Zhejiang, China; E-Mails: nabosun@gmail.com (N.-B.S.); fujianqunshuren@126.com (J.-Q.F.); jinjianzhongshuren@gmail.com (J.-Z.J.)

2 College of Chemical Engineering and Materials Science, Zhejiang University of Technology, Hangzhou 310014, Zhejiang, China; E-Mails: jqweng@zjut.edu.cn (J.-Q.W.); tangchengxia@zjut.edu.cn (C.-X.T.)

* Author to whom correspondence should be addressed; E-Mail: xhliu@zjut.edu.cn; Tel./Fax: +86-571-8832-0832.

Received: 3 September 2013; in revised form: 11 October 2013 / Accepted: 11 October 2013 / Published: 15 October 2013

\begin{abstract}
In order to investigate the biological activity of 1,2,4-triazole compounds, seventeen novel 1,2,4-triazole derivatives containing 1,2,3-thiadiazole moieties were synthesized by multi-step reactions under microwave assisted conditions. The structures were characterized by ${ }^{1} \mathrm{H}-\mathrm{NMR},{ }^{13} \mathrm{C}-\mathrm{NMR}, \mathrm{MS}$ and elemental analyses. The target compounds were evaluated for their in vivo fungicidal activities against Corynespora cassiicola, Pseudomonas syringae pv. Lachrymans, and Pseudoperonospora cubensis, and the results indicated that some of the title compounds displayed good fungicidal activities. Theoretical calculations on the title compounds were carried out at the B3LYP/6-31G (d,p). level. The full geometry optimization was carried out using the 6-31G(d,p) basis set, and the frontier orbital energy, atomic net charges were discussed, and the structure-activity relationships were also studied.
\end{abstract}

Keywords: 1,2,4-triazole; 1,2,3-thiadiazole; synthesis; fungicidal activity; thioether; theoretical calculations 


\section{Introduction}

In recent years, heterocyclic compounds had been receiving considerable attention due to their pharmacological and pesticidal importance [1-9]. Nitrogen-containing heterocycles exhibit excellent biological activities, especially 1,2,4-triazoles and 1,2,3-thiadiazoles. 1,2,4-Triazole rings are typically planar $6 \pi$-electron aromatic systems, featuring an extensive chemistry [10,11]. 1,2,4-Triazole and its derivatives represent one of the most biologically active classes of compounds, displaying a diversity bioactivities in the medicinal and agrochemical field, including anti-inflammatory [12,13], antifungal [14,15], herbicidal [16], antimicrobial [16,17], antiparasitic [18], cytostatic [19], and brassinosteroid biosynthesis inhibitory activities [20]. Some such compounds had been developed as commercial fungicides or herbicides (Figure 1), such as triadimefon, triadimenol, flusilazole, flupoxam and so on.

Figure 1. Commercial drugs and pesticides containing 1,2,4-triazole groups.

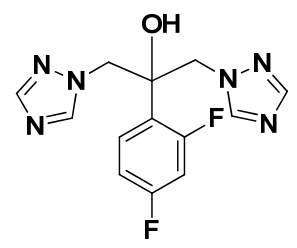

Fluconazole

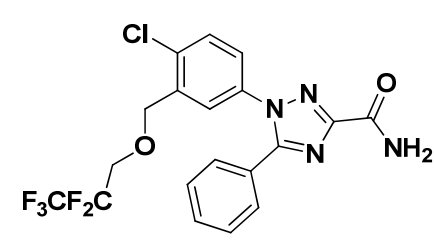

flupoxam

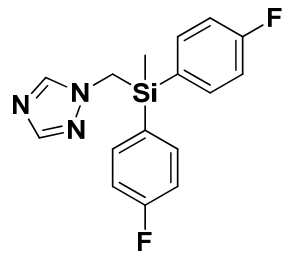

Flusilazole<smiles>CC(C)(C#N)c1cc(Cn2cncn2)cc(C(C)(C)C#N)c1</smiles>

Anastrozole<smiles>CC(C)(C)C(=O)C(Oc1ccc(Cl)cc1)n1cncn1</smiles>

Triadimefon

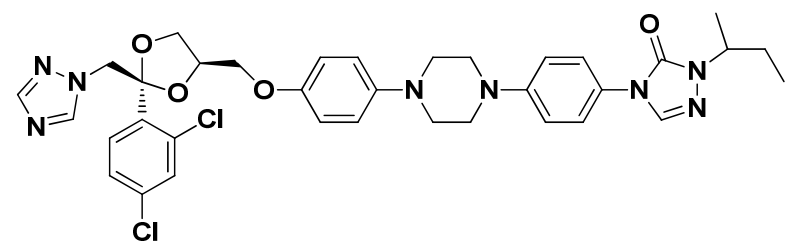

Itraconazole

1,2,3-Thiadiazoles have also been claimed to have beneficial agricultural applications [21,22], because they exhibit wide spectrum biological activities, such as antiviral [23], herbicidal [24,25], antiamoebic [26] and insecticidal [27] activities. For example, after plant inducers such as tiadinil (TDL) and acibenzolar-S-methyl (BTH) (Figure 2) were discovered, 1,2,3-thiadiazole pesticides have become one of the focuses for developing agrochemicals in academia and industry.

Figure 2. Some commercial pesticides containing 1,2,3-thiadiazoles.<smiles>CS(=O)(=O)c1cccc2nnsc12</smiles><smiles>Cc1ccc(NC(=O)c2snnc2C)cc1Cl</smiles>

BTH

tiadinil

There are many reports about each of the two heterocycles, but the combination of 1,2,3-thiadiazole ring with 1,2,4-triazole ring in one molecule is seldom reported either in chemistry or biological 
activity studies. In view of these facts mentioned above, and also as a part of our work [28] on the synthesis of bioactive lead compounds, the title compounds were designed by introducing the 1,2,3-thiadiazole pharmacophore into a 1,2,4-triazole scaffold. Seventeen novel 1,2,4-triazole derivatives were synthesized and characterized by ${ }^{1} \mathrm{H}-\mathrm{NMR},{ }^{13} \mathrm{C}-\mathrm{NMR}$, MS and elemental analysis. The antifungal activities of these compounds were tested in vivo.

\section{Results and Discussion}

\subsection{Chemistry}

The synthesis procedures for title compounds are shown in Scheme 1. The intermediates $\mathbf{1} \sim \mathbf{6}$ were synthesized according our previous method [25]. The intermediate 1 was easily synthesized from diethyl carbonate and $85 \%$ hydrazine hydrate at room temperature. The method of synthesis of the Schiff base 2 was conventional. The key intermediate ethyl 4-methyl-1,2,3-thiadiazole-5-carboxylate (3) was synthesized using the Hurd-Mori method. Then intermediate 3 is reacted with $85 \%$ hydrazine hydrate to give intermediate 4 . The intermediate 5 can be obtained from the intermediate $\mathbf{4}$ and a substituted isothiocyanic ester. The intermediate $\mathbf{5}$ was easily cyclized to give intermediate $\mathbf{6}$ under alkaline conditions, such as $\mathrm{NaOH}$. The intermediate $\mathbf{6}$ is reacted with substituted benzyl chlorides or alkyl chlorides to afford compounds 7. The microwave irradiation assisted synthesis and conventional method were also employed in these experiments. The $\mathrm{NaOH} / \mathrm{DMF} / \mathrm{H}_{2} \mathrm{O}$ system was applied under microwave irradiation. The best reaction conditions were $90{ }^{\circ} \mathrm{C}$ for $15 \mathrm{~min}$ under microwave irradiation. The yield is higher than that of the conventional method, and the reaction time is also shorter (Table 1).

Scheme 1. The synthetic route of title compounds.

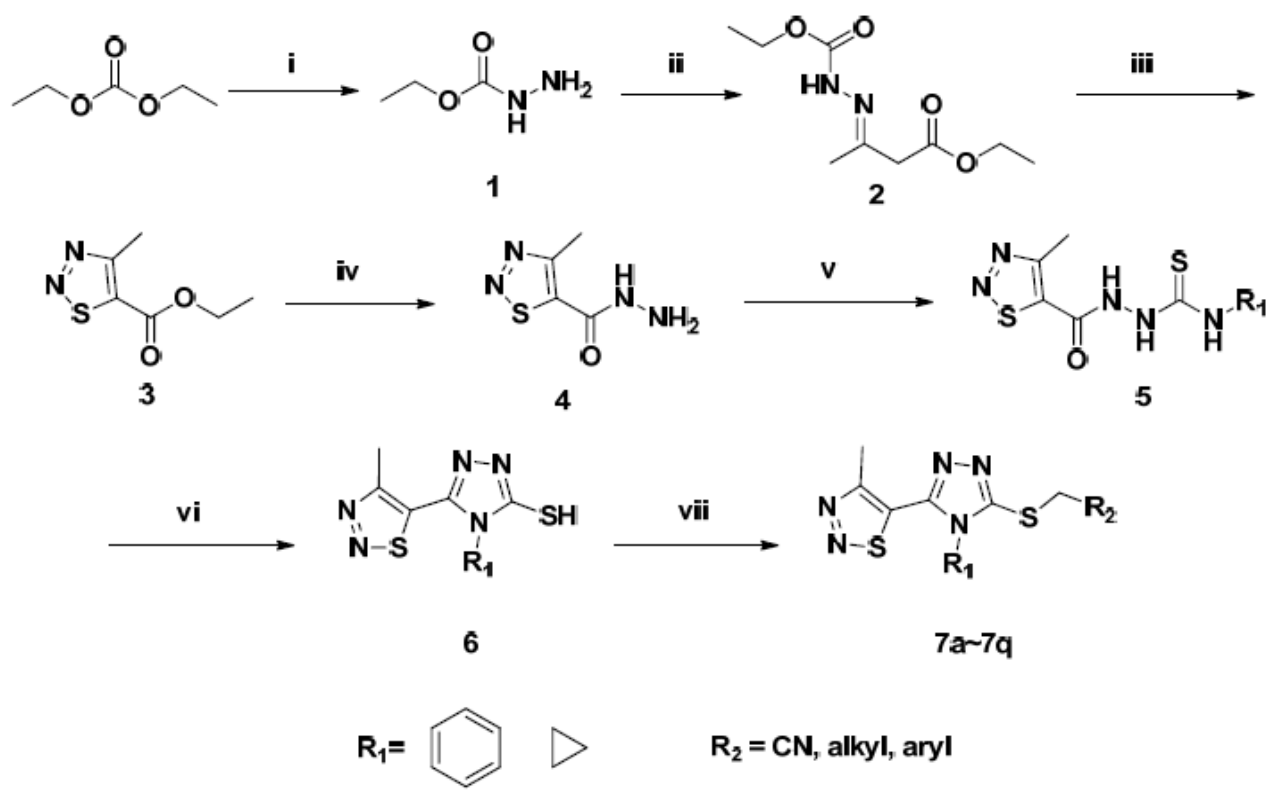

Reagents and conditions: (i) $\mathrm{NH}_{2} \mathrm{NH}_{2} \mathrm{H}_{2} \mathrm{O}$, EtOH, reflux; (ii) ethyl 3-oxobutanoate, EtOH, room temperature; (iii) $\mathrm{SOCl}_{2}$, room temperature; (iv) $\mathrm{NH}_{2} \mathrm{NH}_{2} \mathrm{H}_{2} \mathrm{O}$, EtOH, reflux; (v) $\mathrm{R}_{1} \mathrm{NCS}$, EtOH, reflux; (vi) a. $\mathrm{NaOH}$, reflux; b. $\mathrm{HCl}$; (vii) $\mathrm{R}_{2} \mathrm{CH}_{2} \mathrm{Cl}, \mathrm{K}_{2} \mathrm{CO}_{3}$, DMF, MW. 
Table 1. Comparison of yields of $7 \mathbf{b}$ with or without microwave irradiation.

\begin{tabular}{ccccc}
\hline No. & Method & Time & Temperature $/{ }^{\circ} \mathrm{C}$ & Yield/\% \\
\hline 7b & No-MW & $24 \mathrm{~h}$ & r.t. & 78 \\
7b & MW & $10 \mathrm{~min}$ & 90 & 80 \\
$\mathbf{7 b}$ & MW & $15 \mathrm{~min}$ & 90 & 85 \\
$\mathbf{7 b}$ & MW & $20 \mathrm{~min}$ & 90 & 83 \\
\hline
\end{tabular}

The signal of the $\mathrm{CH}_{2}$ protons of the thioether neighboring the triazole ring was observed at $\delta 4.43 \sim 4.63 \mathrm{ppm}$, respectively. The chemical shifts around $3.0 \mathrm{ppm}$ are from the methyl of the 1,2,3-thiadiazole ring. The ESI-MS spectra showed the $\mathrm{m} / \mathrm{z}$ of the molecular ions, according with their molecular formulas. The elemental analysis results were in accord with the calculated results.

\subsection{Fungicidal Activities and Structure-Activity Relationship (SAR)}

The in vivo fungicidal results of the title compounds against Corynespora cassiicola (CC), Pseudomonas syringae pv. Lachrymans (PS) and Pseudoperonospora cubensis (PC) are listed in Table 2, where iprodione, validamycin and topsin-M were used as controls. As shown in Table 2, lots of the title compounds showed good control efficacy against Corynespora cassiicola at a concentration of $500 \mu \mathrm{g} / \mathrm{mL}$. When the $\mathrm{R}_{1}$ is a phenyl group, only compound $7 \mathrm{e}$ exhibited moderate activity (46.9\%) against Corynespora cassiicola. Notably, compounds $\mathbf{7 b}, \mathbf{7 c}, \mathbf{7 d}$ and $\mathbf{7 e}$ displayed excellent activity $(>80 \%)$ against Corynespora cassiicola, especially compound $\mathbf{7 b}$ which exhibited higher activity than the control. Also the activity of benzene substituted derivatives is higher than that of cyclopropyl substituted ones. Compounds $\mathbf{7 b}, \mathbf{7} \mathbf{c}, \mathbf{7 d}, \mathbf{7 j}, \mathbf{7 k}, \mathbf{7 1}, \mathbf{7 m}$ exhibited fair inhibition effects against Pseudomonas syringae pv. Lachrymans, and the fungicidal activities (control efficacy of 52\%-79\%) were higher than those of iprodione, validamycin and topsin-M. All of them were less effective than the control validamycin. Compound 7 e (71.03\%) and $7 \mathrm{~g}(68.79 \%)$ showed about $70 \%$ control effect against Pseudoperonospora cubensis, at the same level as iprodione, validamycin and topsin-M. In general, the in vivo fungicidal activity of compounds 7 indicated that the substituent changes affect the activity.

In order to further research the structure-activity relationships of the title compounds, some compounds (7o, 7p, 7q) containing cyclopropyl groups synthesized in our other paper [29] were used for comparison. From the data in Table 2, for Corynespora cassiicola, the compounds containing phenyl groups exhibited higher activity than those with cyclopropyl groups, but for Pseudoperonospora cubensis, the results are the opposite, and the compounds containing cyclopropyl groups displayed higher activity than that of phenyl group-containing compounds. For Pseudomonas syringae pv. Lachrymans, the structure-activity relationships are not obvious. Further biological evaluation of all compounds is in progress. The mode of action of title compounds will be explored by molecular modeling. 
Table 2. The antifungal activity of title compounds in vivo.

\begin{tabular}{|c|c|c|c|c|c|}
\hline No. & $\mathbf{R}_{1}$ & $\mathbf{R}_{2}$ & $C C$ & $P S$ & $P C$ \\
\hline $7 a$ & $\mathrm{Ph}$ & $2,4-\mathrm{Cl}_{2} \mathrm{Ph}$ & 72.97 & -1.71 & 50.34 \\
\hline $7 b$ & $\mathrm{Ph}$ & $\mathrm{Ph}$ & 93.19 & 60.67 & 54.51 \\
\hline $7 \mathrm{c}$ & $\mathrm{Ph}$ & 4-F Ph & 82.77 & 55.08 & 44.40 \\
\hline $7 d$ & $\mathrm{Ph}$ & 3-Cl Ph & 82.30 & 53.92 & 39.15 \\
\hline $7 e$ & $\mathrm{Ph}$ & 3-F Ph & 46.90 & 36.71 & 71.03 \\
\hline $7 f$ & $\mathrm{Ph}$ & 2-F Ph & 75.93 & 17.59 & 52.88 \\
\hline $7 g$ & $\mathrm{Ph}$ & 4-MePh & 84.96 & 47.16 & 68.79 \\
\hline $7 \mathrm{~h}$ & $\mathrm{Ph}$ & 4-Et Ph & 70.17 & 11.49 & 19.65 \\
\hline $7 \mathbf{i}$ & $\mathrm{Ph}$ & $\mathrm{CH}_{3} \mathrm{CH}_{2}$ & 75.22 & 48.20 & 42.25 \\
\hline $7 \mathbf{j}$ & $\mathrm{Ph}$ & $2-\mathrm{Cl} \mathrm{Ph}$ & 75.15 & 61.48 & 28.64 \\
\hline $7 \mathbf{k}$ & $\mathrm{Ph}$ & $\mathrm{CN}$ & 75.75 & 67.06 & 26.15 \\
\hline 71 & $\mathrm{Ph}$ & 4-Bu $\mathrm{Ph}$ & 59.92 & 61.00 & 49.38 \\
\hline $7 \mathrm{~m}$ & $\mathrm{Ph}$ & & 70.64 & 63.09 & 18.28 \\
\hline $7 n$ & $\mathrm{Ph}$ & $3,4-\mathrm{Cl}_{2} \mathrm{Ph}$ & 71.07 & 79.76 & 39.35 \\
\hline 70 & & $3-\mathrm{CN} \mathrm{Ph}$ & 28.99 & 24.41 & 69.17 \\
\hline $7 p$ & & & 21.38 & 6.47 & 71.07 \\
\hline $7 q$ & & & 54.03 & 30.17 & 81.62 \\
\hline iprodione & & & 53.52 & 38.49 & 55.92 \\
\hline validamycin & & & 78.20 & -11.42 & 56.31 \\
\hline topsin-M & & & 78.75 & 54.15 & 68.71 \\
\hline
\end{tabular}

(a) CC: Corynespora cassiicola; PS: Pseudomonas syringae pv. Lachrymans; PC: Pseudoperonospora cubensis; (b) The test concentration of antifungal activity is at $500 \mathrm{mg} / \mathrm{mL}$.

\subsection{Molecular Total Energies and Frontier Orbital Energy Analysis}

Molecular total energy and frontier orbital energy levels are listed in Table 3. Energy gap between HOMO and LUMO was calculated by B3LYP.

Table 3. Total energy, frontier orbital energy.

\begin{tabular}{cc}
${\text { DFT }} \\
{$\cline { 2 - 3 }$E_{\text {total }} / \text { Hartree }^{\mathrm{b}}} &{-1765.0575} \\
{E_{\mathrm{HOMO}} / \text { Hartree }} &{-0.221} \\
{E_{\mathrm{LUMO}} / \text { Hartree }} &{-0.069} \\
{\Delta E^{\mathrm{a}} / \text { Hartree }^{\mathrm{a}} \Delta E=E_{\mathrm{LUMO}}-E_{\mathrm{HOMO}} ;^{\mathrm{b}} 1 \text { Hartree }=4.35974417 \times 10^{-18} J=27.2113845 \mathrm{eV} .}$
\end{tabular}

According to the frontier molecular orbital theory, HOMO and LUMO are the most important factors that affect the bioactivity. HOMO has the priority to provide electrons, while LUMO can accept electrons first [30]. Thus study on the frontier orbital energy can provide useful information about the biological mechanism. Taking the DFT result for example, the geometry of the frame of the title compound is hardly influenced by the introduction of either a 1,2,3-thiadiazole ring, 1,2,4-triazole ring, thioether group or phenyl ring (Figure 3). The HOMO of the title compound is mainly located on 
the 1,2,3-thiadiazole ring, 1,2,4-triazole ring and thioether group, while, the LUMO of the title compound is located on the 1,2,3-thiadiazole ring, 1,2,4-triazole ring, thioether group and phenyl ring. The fact that the title compound has strong affinity suggests the importance of the frontier molecular orbital in the $\pi-\pi$ stacking or hydrophobic interactions. This also implies that the orbital interactions between the title compound and the aromatic ring or some other side of residue chains of receptors is dominated by $\pi-\pi$ or hydrophobic interactions among the frontier molecular orbitals.

Figure 3. The HOMO and LUMO of compound $\mathbf{7 b .}$
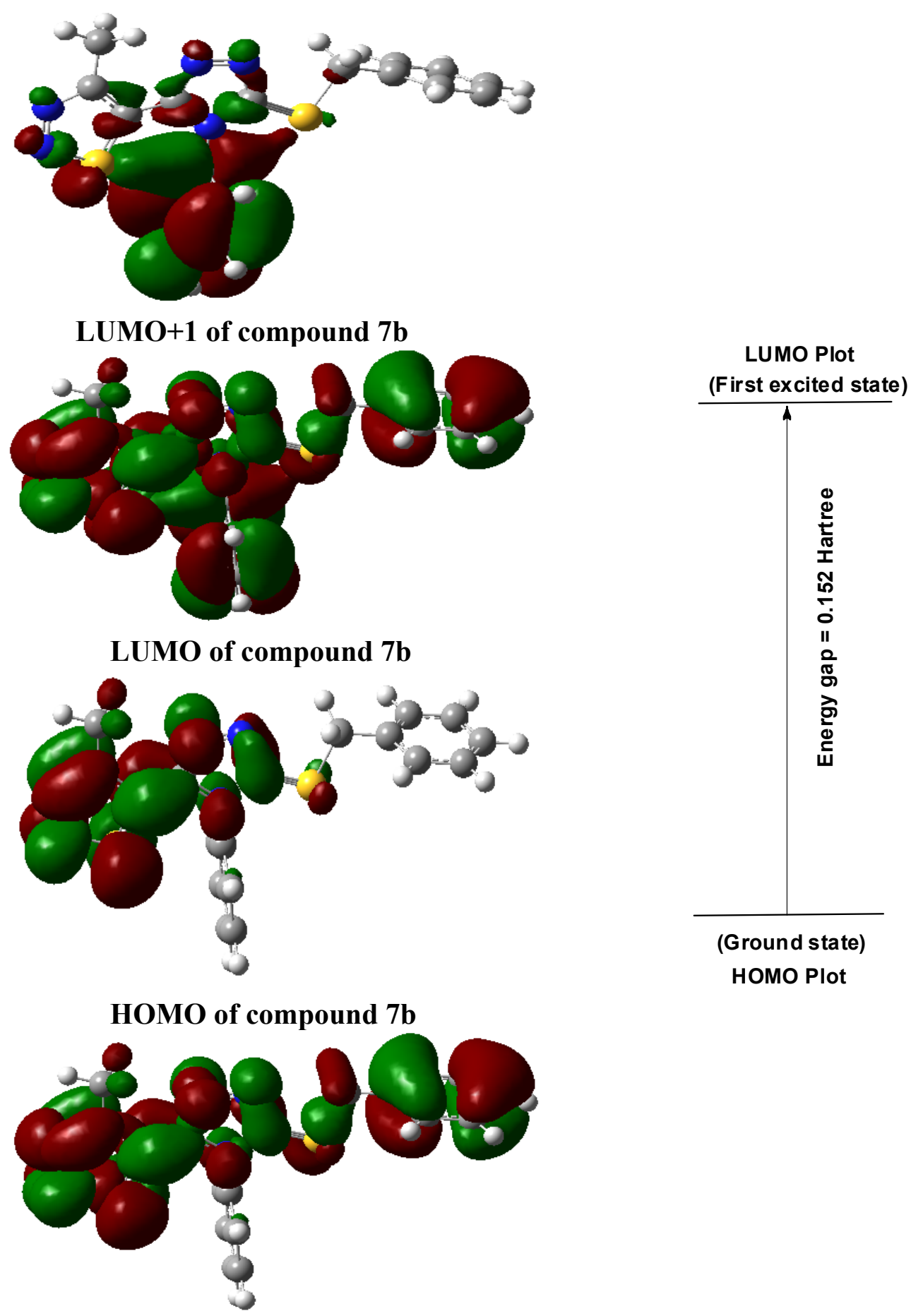

HOMO-1 of compound $7 \mathrm{~b}$ 


\subsection{Mulliken Atomic Charges}

Table 4 lists the calculated Mulliken atomic charges except for the $\mathrm{H}$ atoms. Taking DFT for example again, three atoms $\mathrm{C} 1, \mathrm{C} 2, \mathrm{C} 17, \mathrm{~S} 1$ and $\mathrm{S} 2$ are the most positively charged ones, which can interact with the negative charged part of the receptor easily. The negative charges are mainly located on atoms N1, N2, N3, N4 and N5, so they can interact easily with the positive part of the receptor. Therefore, we supposed that this compound can combine the amino-acid residues on its surface by the interaction of S2-N4-N5 and S1-C1-N3, which may account in part for the bioactivity.

Table 4. Mulliken atomic charges except for $\mathrm{H}$ atoms (e).

\begin{tabular}{cc} 
\\
\multicolumn{2}{c}{}
\end{tabular}




\section{Experimental}

\subsection{Materials and Reagents}

All the reagents were of analytical grade or freshly prepared before use. The monitoring of the progress of all reactions and homogeneity of the synthesized compounds were carried out by thin chromatography (TLC), performed on silica gel plates obtained from Qingdao Ocean Chemicals (Qing Dao, China). Melting points were determined using an X-4 apparatus and are uncorrected. ${ }^{1} \mathrm{H}-\mathrm{NMR}$ and ${ }^{13} \mathrm{C}-\mathrm{NMR}$ spectra were measured on a Bruker AV 400 or 500 instrument using TMS as an internal standard and $\mathrm{CDCl}_{3}$ as the solvent. Mass spectra were recorded on a Thermo Finnigan LCQ Advantage LC/mass detector instrument. Elemental analyses were performed on a Vario EL elemental analyzer. Microwave activation was carried out with a CEM Discover ${ }^{\mathrm{TM}}$ focused microwave $(2,450 \mathrm{MHz}, 300 \mathrm{~W})$.

\subsection{Therotical Calculations}

On the basis of the above structure shown in Scheme 1, compound $7 \mathrm{~b}$ was selected as the initial structure, and the DFT-B3LYP/6-31G $(\mathrm{d}, \mathrm{p})$ [31] methods in the Gaussian 03 package [32] were used to optimize the structure of the title compound. Vibration analysis showed that the optimized structures were in accordance with the minimum points on the potential energy surfaces. All the convergent precisions were the system default values, and all the calculations were carried out on a personal computer.

\subsection{Chemical Synthesis}

\subsubsection{Synthesis of Intermediates}

\subsubsection{Preparation of 4-methyl-1,2,3-thiadiazole-5-carbohydrazide (compounds 1 4)}

The synthetic route is shown in Scheme 1. The intermediate 4-methyl-1,2,3-thiadiazole-5carboxylic acid hydrazide (2) was synthesized according to the literature [25]. Carbonic acid diethyl ester $(11.8 \mathrm{~g}, 0.1 \mathrm{~mol})$ and hydrazine hydrate $(5.6 \mathrm{~mL}, 0.095 \mathrm{~mol}, 85 \%)$ were added into a $250 \mathrm{~mL}$ round-bottom flask equipped with a condenser. The reaction mixture was heated to $50{ }^{\circ} \mathrm{C}$ and stirred for $20 \mathrm{~min}$, and then cooled down to room temperature and further stirred for $30 \mathrm{~h}$. Water, ethanol, and excess carbonic acid diethyl ester were distilled off under reduced pressure. After drying, compound (2) (9.88 g, 95\% yield) was obtained as white crystals. To a stirred solution of compound 2 (6.36 g, $0.06 \mathrm{~mol})$ in ethanol $(16.7 \mathrm{~mL})$, a solution of ethyl acetoacetate $(7.8 \mathrm{~g}, 0.06 \mathrm{~mol})$ in ethanol $(3.7 \mathrm{~mL})$ was added at room temperature. Stirring was continued for $6 \mathrm{~h}$. Then, the solvent was removed in vacuo and the crude 3-ethoxycarbonyl hydrazonoacetic acid ethyl ester was directly used in the next step without further purification. 3-Ethoxycarbonyl hydrazonoacetic acid ethyl ester (12.8 g, $0.06 \mathrm{~mol})$ was dissolved in dry dichloromethane $(25 \mathrm{~mL})$, and thionyl chloride $(20 \mathrm{~mL})$ was added to the stirred reaction mixture dropwise at $0{ }^{\circ} \mathrm{C}$ for $1 \mathrm{~h}$. Next, the reaction mixture was allowed to stand for $20 \mathrm{~h}$ at room temperature. The excess thionyl chloride and dichloromethane were distilled off, and the remaining residue was subjected to fractional distillation under reduced pressure. A slightly yellowish oil of 3 (7.95 g, 77\% yield) was obtained. A mixture of 4-methyl-1,2,3-thiadiazole-5-carboxylic acid 
ethyl ester $(1.72 \mathrm{~g}, 10 \mathrm{mmol})$ and hydrazine hydrate $(12 \mathrm{mmol})$ in $10 \mathrm{~mL}$ of methanol was stirred vigorously for $0.5 \mathrm{~h}$ at room temperature, the resulting mixture was then filtered, and the solid was washed with cold methanol. After drying, the solid was recrystallized from methanol to give intermediate (4).

\subsubsection{2-(4-Methyl-1,2,3-thiadiazole-5-carbonyl)-N-phenylhydrazinecarbothioamide (5)}

A mixture of 4-methyl-1,2,3-thiadiazole-5-carbohydrazide and isothiocyanatobenzene was refluxed for $3 \mathrm{~h}$ in ethanol. After cooling down to room temperature, the product obtained was recrystallized from methanol to give 5.

\subsubsection{5-(4-Methyl-1,2,3-thiadiazol-5-yl)-4-phenyl-4H-1,2,4-triazole-3-thiol (6)}

A mixture of compound $5(10 \mathrm{mmol})$ in aqueous $\mathrm{NaOH}$ solution $(5 \mathrm{~mL}, 2 \mathrm{~N})$ was refluxed for $4 \mathrm{~h}$. After cooling down to room temperature, $\mathrm{HCl}$ aqueous solution $(4 \mathrm{~N})$ was added to afford a large amount of precipitate. The solid was filtered, dried and recrystallized from methanol to give intermediate 6.

\subsubsection{General Procedure for the Preparation of Thioethers 7}

A CEM-designed $10 \mathrm{~mL}$ pressure-rated vial was charged with DMF (15 mL), 6 (1.5 g, $5.1 \mathrm{mmol})$ and $\mathrm{K}_{2} \mathrm{CO}_{3}(0.2 \mathrm{~g}, 5.6 \mathrm{mmol})$. The mixture was irradiated in a CEM Discover Focused Synthesizer $\left(150 \mathrm{w}, 90{ }^{\circ} \mathrm{C}, 200 \mathrm{psi}, 15\right.$ minutes). The mixture was cooled to room temperature by passing compressed air through the microwave cavity for 2 minutes. It was poured into cold ice $(40 \mathrm{~mL})$ and the precipitate formed was filtered. The crude solid was recrystallized from $\mathrm{EtOH}$ to give the title compound 7a. All the other compounds are synthesized according to the same procedure.

5-(5-((2,4-Dichlorobenzyl)thio)-4-phenyl-4H-1,2,4-triazol-3-yl)-4-methyl-1,2,3-thiadiazole (7a). White crystals, yield 74\%, m.p. 102-103 ${ }^{\circ} \mathrm{C} ;{ }^{1} \mathrm{H}-\mathrm{NMR}\left(\mathrm{CDCl}_{3}, 400 \mathrm{MHz}\right)$ 8: 3.07 (s, 3H, Het-Me), 4.61 (s, 2H, $\left.\mathrm{SCH}_{2}\right), 7.14-7.22(\mathrm{~m}, 4 \mathrm{H}, \mathrm{ArH}), 7.35-7.40$ (m, 2H, ArH), 7.56-7.60 (m, 1H, ArH), 7.64-7.66 (m, 1H, ArH). MS (ESI), $m / z$ : $435(\mathrm{M}+1)^{+}$. Elemental anal. (\%), calculated: C, 49.77; H, 3.02; N, 16.12; found: C, 49.98; H, 3.12; N,16.09.

5-(5-Benzylthio)-4-phenyl-4H-1,2,4-triazol-3-yl)-4-methyl-1,2,3-thiadiazole (7b). White crystals, yield 75\%, m.p. $120-121{ }^{\circ} \mathrm{C} ;{ }^{1} \mathrm{H}-\mathrm{NMR}\left(\mathrm{CDCl}_{3}, 400 \mathrm{MHz}\right)$ 8: 3.07 (s, 3H, Het-Me), 4.63 (s, 2H, SCH$), 7.08$ (d, $J=7.99 \mathrm{~Hz}, 2 \mathrm{H}, \mathrm{ArH}), 7.28-7.32$ (m, 3H, ArH), 7.36-7.38 (m, 2H, ArH), 7.54 (t, $J=8.00 \mathrm{~Hz}, 2 \mathrm{H}$, ArH), $7.64(\mathrm{t}, J=7.38 \mathrm{~Hz}, 1 \mathrm{H}, \mathrm{ArH})$. MS (ESI), $m / z: 366(\mathrm{M}+1)^{+}$. Elemental anal. (\%), calculated: C, 59.15; H, 4.14; N, 19.16; found: C, 58.89; H, 4.43; N,19.32.

5-(5-((4-Fluorobenzyl)thio)-4-phenyl-4H-1,2,4-triazol-3-yl)-4-methyl-1,2,3-thiadiazole (7c). White crystals, yield 82\%, m.p. 88-89 ${ }^{\circ} \mathrm{C}$; ${ }^{1} \mathrm{H}-\mathrm{NMR}\left(\mathrm{CDCl}_{3}, 400 \mathrm{MHz}\right) \delta$ : 3.06 (s, 3H, Het-Me), 4.51 (s, $\left.2 \mathrm{H}, \mathrm{SCH}_{2}\right)$, 6.97 (d, $J=8.65 \mathrm{~Hz}, 2 \mathrm{H}, \operatorname{ArH}), 7.13(\mathrm{~d}, J=7.30 \mathrm{~Hz}, 2 \mathrm{H}, \operatorname{ArH}), 7.36-7.39$ (m, 2H, ArH), 7.57 (t, $J=8.05 \mathrm{~Hz}, 2 \mathrm{H}, \mathrm{ArH}), 7.65$ (t, $J=7.47 \mathrm{~Hz}, 1 \mathrm{H}, \mathrm{ArH})$. MS (ESI), $m / z: 384(\mathrm{M}+1)^{+}$. Elemental anal. (\%), calculated: C, 56.38; H, 3.68; N, 18.26; found: C, 56.12; H, 3.43; N,18.32. 
5-(5-((3-Chlorobenzyl)thio)-4-phenyl-4H-1,2,4-triazol-3-yl)-4-methyl-1,2,3-thiadiazole (7d). White crystals, yield 86\%, m.p. 101-102 ${ }^{\circ} \mathrm{C}$; ${ }^{1} \mathrm{H}-\mathrm{NMR}\left(\mathrm{CDCl}_{3}, 400 \mathrm{MHz}\right)$ 8: 3.04 (s, 3H, Het-Me), 4.47 (s, 2H, $\left.\mathrm{SCH}_{2}\right), 7.10(\mathrm{~d}, J=7.60 \mathrm{~Hz}, 2 \mathrm{H}, \mathrm{ArH}), 7.21-7.28(\mathrm{~m}, 3 \mathrm{H}, \mathrm{ArH}), 7.36(\mathrm{~s}, 1 \mathrm{H}, \mathrm{ArH}), 7.56(\mathrm{t}$, $J=7.25 \mathrm{~Hz}, 2 \mathrm{H}, \mathrm{ArH}), 7.65$ (t, $J=7.18 \mathrm{~Hz}, 1 \mathrm{H}, \mathrm{ArH})$. MS (ESI), $m / z$ : $400(\mathrm{M}+1)^{+}$. Elemental anal. (\%), calculated: C, 54.06; H, 3.53; N, 17.51; found: C, 54.33; H, 3.63; N,17.67.

5-(5-((3-Fluorobenzyl)thio)-4-phenyl-4H-1,2,4-triazol-3-yl)-4-methyl-1,2,3-thiadiazole (7e). White crystals, yield 88\%, m.p. 98-99 ${ }^{\circ} \mathrm{C}$; ${ }^{1} \mathrm{H}-\mathrm{NMR}\left(\mathrm{CDCl}_{3}, 400 \mathrm{MHz}\right)$ 8: 3.06 (s, 3H, Het-Me), $4.52\left(\mathrm{~s}, 2 \mathrm{H}, \mathrm{SCH}_{2}\right)$, $6.97(\mathrm{t}, J=8.25 \mathrm{~Hz}, 1 \mathrm{H}, \mathrm{ArH}), 7.09-7.24(\mathrm{~m}, 4 \mathrm{H}, \mathrm{ArH}), 7.56$ (t, $J=7.86 \mathrm{~Hz}, 2 \mathrm{H}, \mathrm{ArH}), 7.63-7.69$ (m, 2H, ArH). MS (ESI), $m / z: 384(\mathrm{M}+1)^{+}$. Elemental anal. (\%), calculated: C, 56.38; H, 3.68; N, 18.26; found: C, 56.75; H, 3.63; N,18.49.

5-(5-((2-Fluorobenzyl)thio)-4-phenyl-4H-1,2,4-triazol-3-yl)-4-methyl-1,2,3-thiadiazole (7f). White crystals, yield 78\%, m.p. ${ }^{104-105}{ }^{\circ} \mathrm{C} ;{ }^{1} \mathrm{H}-\mathrm{NMR}\left(\mathrm{CDCl}_{3}, 400 \mathrm{MHz}\right) \delta$ : 3.05 (s, 3H, Het-Me), 4.50 (s, 2H, $\left.\mathrm{SCH}_{2}\right), 7.05$ (d, J=7.70 Hz, 2H, ArH), 7.17-7.32 (m, 4H, ArH), 7.52 (t, J=8.09 Hz, 2H, ArH), $7.61(\mathrm{t}, J=6.97 \mathrm{~Hz}, 1 \mathrm{H}, \mathrm{ArH})$. MS (ESI), $m / z: 384(\mathrm{M}+1)^{+}$. Elemental anal. (\%), calculated: C, 56.38; H, 3.68; N, 18.26; found: C, 56.13; H, 3.77; N,18.34.

4-Methyl-5-(5-((4-methylbenzyl)thio)-4-phenyl-4H-1,2,4-triazol-3-yl)-1,2,3-thiadiazole (7g). White crystals, yield 86\%, m.p. $115-116{ }^{\circ} \mathrm{C}$; ${ }^{1} \mathrm{H}-\mathrm{NMR}\left(\mathrm{CDCl}_{3}, 400 \mathrm{MHz}\right) \delta: 2.33$ (s, 3H, Ar-Me), 3.06 (s, 3H, Het-Me), 4.55 (s, 2H, $\left.\mathrm{SCH}_{2}\right), 7.02$ (d, $\left.J=7.31 \mathrm{~Hz}, 2 \mathrm{H}, \mathrm{ArH}\right), 7.05-7.22(\mathrm{~m}, 3 \mathrm{H}, \mathrm{ArH}), 7.29$ (d, $J=7.45 \mathrm{~Hz}, 1 \mathrm{H}$, ArH), 7.52 (t, $J=7.98 \mathrm{~Hz}, 2 \mathrm{H}, \operatorname{ArH}), 7.62$ (t, $J=7.47 \mathrm{~Hz}, 1 \mathrm{H}, \operatorname{ArH})$. MS (ESI), $m / z: 380(\mathrm{M}+1)^{+}$. Elemental anal. (\%), calculated: C, 60.13; H, 4.52; N, 18.45; found: C, 59.89; H, 4.67; N,18.60.

4-Methyl-5-(5-((4-ethylbenzyl)thio)-4-phenyl-4H-1,2,4-triazol-3-yl)-1,2,3-thiadiazole (7h). White crystals, yield 89\%, m.p. $135-136{ }^{\circ} \mathrm{C} ;{ }^{1} \mathrm{H}-\mathrm{NMR}\left(\mathrm{CDCl}_{3}, 400 \mathrm{MHz}\right) \delta: 1.28(\mathrm{t}, 3 \mathrm{H}, \mathrm{Me}), 2.63(\mathrm{q}, 2 \mathrm{H}$, Ar- $\mathrm{CH}_{2}$ ), 3.06 (s, 3H, Het-Me), 4.55 (s, 2H, $\mathrm{SCH}_{2}$ ), 7.06-7.21 (m, 5H, ArH), 7.51-7.62 (m, 4H, ArH). MS (ESI), $m / z: 394(\mathrm{M}+1)^{+}$. Elemental anal. (\%), calculated: C, 61.04; H, 4.87; N, 17.80; found: C, $60.99 ; \mathrm{H}, 4.97 ; \mathrm{N}, 17.56$.

4-Methyl-5-(4-phenyl-5-(propylthio)-4H-1,2,4-triazol-3-yl)-1,2,3-thiadiazole (7i). White crystals, yield 59\%, m.p. $106-107^{\circ} \mathrm{C} ;{ }^{1} \mathrm{H}-\mathrm{NMR}\left(\mathrm{CDCl}_{3}, 400 \mathrm{MHz}\right) \delta: 1.03(\mathrm{t}, J=7.37 \mathrm{~Hz}, 3 \mathrm{H}, \mathrm{Me}), 1.79-1.86(\mathrm{~m}$, $2 \mathrm{H}, \mathrm{CH}_{3} \mathrm{CH}_{2}$ ), 3.06 (s, 3H, Het-Me), 3.30 (t, $\left.J=7.17 \mathrm{~Hz}, 3 \mathrm{H}, \mathrm{CH}_{3} \mathrm{CH}_{2} \mathrm{CH}_{2}\right), 4.55$ (s, 2H, $\mathrm{SCH}_{2}$ ), 7.23-7.25 (m, 2H, ArH), 7.58-7.68 (m, 3H, ArH). MS (ESI), m/z: $318(\mathrm{M}+1)^{+}$. Elemental anal. (\%), calculated: C, 52.97; H, 4.76; N, 22.06; found: C, 52.76; H, 4.67; N,21.96.

5-(5-((3-Chlorobenzyl)thio)-4-phenyl-4H-1,2,4-triazol-3-yl)-4-methyl-1,2,3-thiadiazole (7j). White crystals, yield 85\%, m.p. $138-139{ }^{\circ} \mathrm{C} ;{ }^{1} \mathrm{H}-\mathrm{NMR}\left(\mathrm{CDCl}_{3}, 400 \mathrm{MHz}\right) \delta$ : 3.06 (s, 3H, Het-Me), 4.49 (s, 2H, $\left.\mathrm{SCH}_{2}\right)$, $7.10(\mathrm{~d}, J=7.16 \mathrm{~Hz}, 2 \mathrm{H}, \mathrm{ArH}), 7.22-7.25$ (m, 1H, ArH), 7.27-7.29 (m, 1H, ArH), 7.37 (s, 1H, ArH), $7.57(\mathrm{t}, J=8.00 \mathrm{~Hz}, 2 \mathrm{H}, \mathrm{ArH}), 7.66(\mathrm{~d}, J=7.58 \mathrm{~Hz}, 1 \mathrm{H}, \mathrm{ArH})$. MS (ESI), $m / z: 400(\mathrm{M}+1)^{+}$. Elemental anal. (\%), calculated: C, 54.06; H, 3.53; N, 17.51; found: C, 53.89; H, 3.55; N,17.78.

2-((5-(4-Methyl-1,2,3-thiadiazol-5-yl)-4-phenyl-4H-1,2,4-triazol-3-yl)thio)acetonitrile (7k). White crystals, yield 84\%, m p. $118-119{ }^{\circ} \mathrm{C} ;{ }^{1} \mathrm{H}-\mathrm{NMR}\left(\mathrm{CDCl}_{3}, 500 \mathrm{MHz}\right) \delta$ : 3.08 (s, 3H, Het-Me), $4.16\left(\mathrm{~s}, 2 \mathrm{H}, \mathrm{SCH}_{2}\right)$, 
$7.28(\mathrm{~d}, J=7.7 \mathrm{~Hz}, 1 \mathrm{H}, \mathrm{ArH}), 7.34(\mathrm{~d}, J=7.7 \mathrm{~Hz}, 1 \mathrm{H}, \mathrm{ArH}), 7.63-7.73(\mathrm{~m}, 3 \mathrm{H}, \mathrm{ArH}) .{ }^{13} \mathrm{C}-\mathrm{NMR}\left(\mathrm{CDCl}_{3}\right.$, $125 \mathrm{MHz}) \delta: 14.8,17.8,115.1,127.8,130.6,131.7,132.2,148.8,150.7,160.4$. MS (ESI), $m / z: 315$ $(\mathrm{M}+1)^{+}$. Elemental anal. (\%), calculated: C, 49.66; H, 3.21; N, 26.73; found: C, 49.78; H, 3.11; N,26.55.

5-(5-((4-(tert-Butyl)benzyl)thio)-4-phenyl-4H-1,2,4-triazol-3-yl)-4-methyl-1,2,3-thiadiazole (7l). White crystals, yield $86 \%$, m.p. $125-126{ }^{\circ} \mathrm{C} ;{ }^{1} \mathrm{H}-\mathrm{NMR}\left(\mathrm{CDCl}_{3}, 500 \mathrm{MHz}\right) \delta$ : 1.31 (s, 9H, Bu), 3.07 (s, 3H, Het-Me), 4.52 (s, 2H, SCH ), 7.09 (d, $J=8.0 \mathrm{~Hz}, 2 \mathrm{H}, \mathrm{ArH}), 7.29$ (d, $J=8.5 \mathrm{~Hz}, 2 \mathrm{H}, \mathrm{ArH}), 7.33$ (d, $J=8.5 \mathrm{~Hz}, 2 \mathrm{H}, \mathrm{ArH}), 7.54(\mathrm{t}, J=8.1 \mathrm{~Hz}, 2 \mathrm{H}, \mathrm{ArH}), 7.63(\mathrm{~d}, J=7.5 \mathrm{~Hz}, 1 \mathrm{H}, \mathrm{ArH}) .{ }^{13} \mathrm{C}-\mathrm{NMR}\left(\mathrm{CDCl}_{3}\right.$, $125 \mathrm{MHz}) \delta$ : 14.8, 31.3, 34.6, 36.9, 125.7, 127.9, 128.9, 130.5, 131.5, 132.1, 132.9, 133.7, 147.6, 151.1, 154.3, 159.9. MS (ESI), $m / z: 423(\mathrm{M}+1)^{+}$. Elemental anal. (\%), calculated: C, 62.68; H, 5.50; N, 16.61; found: C, 62.77; H, 5.55; N,16.45.

5-(5-(((2-Chlorothiazol-5-yl)methyl)thio)-4-phenyl-4H-1,2,4-triazol-3-yl)-4-methyl-1,2,3-thiadiazole (7m). White crystals, yield 89\%, m.p. ${ }^{129-130}{ }^{\circ} \mathrm{C} ;{ }^{1} \mathrm{H}-\mathrm{NMR}\left(\mathrm{CDCl}_{3}, 500 \mathrm{MHz}\right) \delta: 3.08$ (s, 3H, Het-Me), $4.68\left(\mathrm{~s}, 2 \mathrm{H}, \mathrm{SCH}_{2}\right), 7.10(\mathrm{~d}, J=7.8 \mathrm{~Hz}, 2 \mathrm{H}, \mathrm{ArH}), 7.52(\mathrm{~s}, 1 \mathrm{H}$, thiazole-H), $7.61(\mathrm{t}, J=8.0 \mathrm{~Hz}, 2 \mathrm{H}$, $\mathrm{ArH}), 7.70$ (t, $J=7.5 \mathrm{~Hz}, 1 \mathrm{H}, \mathrm{ArH}) .{ }^{13} \mathrm{C}-\mathrm{NMR}\left(\mathrm{CDCl}_{3}, 125 \mathrm{MHz}\right) \delta: 14.8,28.3,127.8,130.8,131.7$, 131.9, 133.3, 136.4, 140.9, 148.2, 152.3, 152.9, 160.1. MS (ESI), $m / z$ : $408(\mathrm{M}+1)^{+}$. Elemental anal. (\%), calculated: C, 44.27; H, 2.72; N, 20.65; found: C, 44.44; H, 2.81; N,20.77.

5-(5-((3,4-Dichlorobenzyl)thio)-4-phenyl-4H-1,2,4-triazol-3-yl)-4-methyl-1,2,3-thiadiazole

$(7 n)$.

White crystals, yield 81\%, m.p. ${ }^{109-110}{ }^{\circ} \mathrm{C} ;{ }^{1} \mathrm{H}-\mathrm{NMR}\left(\mathrm{CDCl}_{3}, 500 \mathrm{MHz}\right) \delta: 3.06$ (s, 3H, Het-Me), 4.49 (s, 2H, $\left.\mathrm{SCH}_{2}\right), 7.14$ (d, J=7.7 Hz, 2H, ArH), 7.30 (s, 1H, ArH), 7.44 (d, J=8.3 Hz, 2H, ArH), 7.57 (t, $J=8.0 \mathrm{~Hz}, 2 \mathrm{H}, \mathrm{ArH}), 7.66$ (d, $J=7.5 \mathrm{~Hz}, 1 \mathrm{H}, \mathrm{ArH})$. MS (ESI), $m / z$ : $423(\mathrm{M}+1)^{+}$. Elemental anal. (\%), calculated: C, 49.77; H, 3.02; N, 16.12; found: C, 49.58; H, 3.35; N,16.01.

3-(((4-Cyclopropyl-5-(4-methyl-1,2,3-thiadiazol-5-yl)-4H-1,2,4-triazol-3-yl)thio) methyl) benzonitrile (7o). White solid, yield 82\%, m.p. ${ }^{133-134}{ }^{\circ} \mathrm{C}$; ${ }^{1} \mathrm{H}-\mathrm{NMR}$ (CDCl3, $\left.400 \mathrm{MHz}\right)$ 8: 0.79-0.83 (m, 2H, cyclopropane- $\left.\mathrm{CH}_{2}\right), 1.13-1.18\left(\mathrm{~m}, 2 \mathrm{H}\right.$, cyclopropane- $\left.\mathrm{CH}_{2}\right), 2.95\left(\mathrm{~s}, 3 \mathrm{H}, \mathrm{CH}_{3}\right), 2.97-3.02(\mathrm{~m}, 1 \mathrm{H}$, cyclopropane-CH), $4.53\left(\mathrm{~s}, 2 \mathrm{H}, \mathrm{CH}_{2}\right), 7.37$ (t, J=7.22 Hz, 1H, Ph-H), 7.51 (d, J=7.22 Hz, 1H, Ph-H), $7.71(\mathrm{t}, J=7.84 \mathrm{~Hz}, 2 \mathrm{H}, \mathrm{Ph}-\mathrm{H})$; MS (ESI), $m / z$ : $355(\mathrm{M}+1)^{+}$; Elemental analysis for $\mathrm{C}_{16} \mathrm{H}_{14} \mathrm{~N}_{6} \mathrm{~S}_{2}$ : found C 54.21, H 3.70, N 23.99; calc. C, 54.22; H, 3.98; N, 23.71.

5-(4-Cyclopropyl-5-(prop-2-yn-1-ylthio)-4H-1,2,4-triazol-3-yl)-4-methyl-1,2,3-thiadiazole (7p). White solid, yield 77\%, m.p. $107-108{ }^{\circ} \mathrm{C} ;{ }^{1} \mathrm{H} \mathrm{NMR}\left(\mathrm{CDCl}_{3}, 400 \mathrm{MHz}\right) \delta$ : 0.78-0.94 (m, 2H, cyclopropane- $\left.\mathrm{CH}_{2}\right)$, 1.17-1.21 (m, 2H, cyclopropane- $\left.\mathrm{CH}_{2}\right), 2.24(\mathrm{~s}, 1 \mathrm{H}, \mathrm{CH}), 2.95\left(\mathrm{~s}, 3 \mathrm{H}, \mathrm{CH}_{3}\right), 2.99-3.11(\mathrm{~m}, 1 \mathrm{H}$, cyclopropane-CH), 4.09 (s, 2H, CH$)_{2}$.MS (ESI): $m / z 278(\mathrm{M}+1)^{+}$; Elemental analysis for $\mathrm{C}_{11} \mathrm{H}_{11} \mathrm{~N}_{5} \mathrm{~S}_{2}$ : found C 47.88, H 4.23, N 25.14; calc. C, 47.63; H, 4.00; N, 25.25.

5-(4-Cyclopropyl-5-(octylthio)-4H-1,2,4-triazol-3-yl)-4-methyl-1,2,3-thiadiazole (7q). White solid, yield $81 \%$, m.p. $67-68{ }^{\circ} \mathrm{C}$; ${ }^{1} \mathrm{H}-\mathrm{NMR}\left(\mathrm{CDCl}_{3}, 400 \mathrm{MHz}\right) \delta: 0.79-0.86\left(\mathrm{~m}, 5 \mathrm{H}\right.$, cyclopropane- $\mathrm{CH}_{2}$ and $\left.\mathrm{CH}_{2}\right), 1.14-1.24\left(\mathrm{~m}, 11 \mathrm{H}\right.$, cyclopropane- $\mathrm{CH}_{2}$ and $\left.\mathrm{CH}_{2}\right), 1.36-1.44\left(\mathrm{~m}, 2 \mathrm{H}, \mathrm{CH}_{2}\right), 1.74-1.81\left(\mathrm{~m}, 2 \mathrm{H}, \mathrm{CH}_{2}\right)$, 2.95(s, 3H, $\left.\mathrm{CH}_{3}\right), 2.99-3.05\left(\mathrm{~m}, 1 \mathrm{H}\right.$, cyclopropane-CH), 3.33 (t, 3H, $\left.\mathrm{CH}_{3}\right)$; MS (ESI) m/z: $352(\mathrm{M}+1)^{+}$; Elemental analysis for $\mathrm{C}_{16} \mathrm{H}_{25} \mathrm{~N}_{5} \mathrm{~S}_{2}$ : found C 54.86, H 7.24, N 20.20; calc. C, 54.67; H, 7.17; N, 19.92. 


\subsection{Fungicidal Activities}

Fungicidal activity of compounds $7 \mathbf{a}-\mathbf{q}$ against Pseudoperonospora cubensis(PC), Corynespora cassiicola $(C C)$, Pseudomonas syringae pv. Lachrymans(PS) were evaluated according to reference [11], and a potted plant test method was adopted. Germination was conducted by soaking cucumber seeds in water for $2 \mathrm{~h}$ at $50{ }^{\circ} \mathrm{C}$ and then keeping the seeds moist for $24 \mathrm{~h}$ at $28{ }^{\circ} \mathrm{C}$ in an incubator. When the radicles were $0.5 \mathrm{~cm}$, the seeds were grown in plastic pots containing a 1:1 $(\mathrm{v} / \mathrm{v})$ mixture of vermiculite and peat. Cucumber plants used for inoculations were at the stage of two seed leaves. Tested compounds and commercial fungicides were sprayed with a hand spray on the surface of the seed leaves on a fine morning, at the standard concentration of $500 \mu \mathrm{g} / \mathrm{mL}$, iprodione, validamycin and topsin-M were used as a control. After $2 \mathrm{~h}$, inoculations of Pseudoperonospora cubensis, Corynespora cassiicola were carried out by spraying a conidial suspension, inoculation of Pseudomonas syringae pv. Lachrymans was carried out by spraying fungal suspension. The experiment was repeated 4 times. After inoculation, the plants were maintained at $18-30{ }^{\circ} \mathrm{C}$ [mean temperature of $24{ }^{\circ} \mathrm{C}$ and above $80 \%$ relative humidity $(\mathrm{RH})]$. The fungicidal activity were evaluated when the nontreated cucumber plant (blank) fully developed symptoms. The area of inoculated treated leaves covered by disease symptoms was assessed and compared to that of nontreated ones to determine the average disease index. The relative control efficacy of compounds compared to the blank assay was calculated via the following equation:

$$
\text { relative control efficacy }(\%)=(C K-P T) / C K \times 100 \%
$$

where CK is the average disease index during the blank assay and PT is the average disease index after treatment during testing.

\section{Conclusions}

In summary, a series of 1,2,4-triazole derivatives containing 1,2,3-thiadiazole rings were synthesized in good yields. The preliminary bioassays showed that some of the compounds had good fungicidal activity. The present findings provided a powerful complement to the SARs of fungicides, and warrant future investigation of the mechanism of action of these analogues.

\section{Acknowledgments}

The project was supported by Applied Chemistry Foundation of Zhejiang Shuren University (YH2013G02, YH2012S09, YH2013X14) and Zhejiang Provincial Natural Science Foundation of China (LQ13B020003), National Natural Science Foundation of China (No. 21002090), The Key Innovation Team of Science and Technology in Zhejiang Province (2010R50018-06) and the National Key Technologies R\&D Program (2011BAE06B03-01). The fungicidal activity was determined by Li Bao-Ju Group, Institute of Vegetables and Flowers, Chinese Academy of Agricultural Sciences, Beijing, China.

\section{Conflicts of Interest}

The authors declare no conflict of interest 


\section{References}

1. Liu, X.H.; Tan, C.X.; Weng, J.Q. Phase transfer-catalyzed, One-pot synthesis of some novel n-pyrimidinyl-n'-nicotinyl thiourea derivatives. Phosphorus, Sulfur Silicon Relat. Elem. 2011, $186,552-557$.

2. Su, N.N.; Li, Y.; Yu, S.J.; Zhang, X.; Liu, X.H.; Zhao, W.G. Microwave-assisted synthesis of some novel 1,2,3-triazoles by click chemistry, and their biological activity. Res. Chem. Intermed. 2013, 39, 759-766.

3. Liu, X.H.; Pan, L.; Tan, C.X.; Weng, J.Q.; Wang, B.L.; Li, Z.M. Synthesis, crystal structure, bioactivity and DFT calculation of new oxime ester derivatives containing cyclopropane moiety. Pestic. Biochem. Physiol. 2011, 101, 143-147.

4. Liu, X.H.; Pan, L.; Ma, Y.; Weng, J.Q.; Tan, C.X.; Li, Y.H.; Shi, Y.X.; Li, B.J.; Li, Z.M.; Zhang, Y.G. Design, synthesis, biological activities, and 3D-QSAR of new N,N'-diacylhydrazines containing 2-(2,4-dichlorophenoxy)propane moiety. Chem. Biol. Drug Des. 2011, 78, 689-694.

5. Chen, P.Q.; Tan, C.X.; Weng, J.Q.; Liu, X.H. Synthesis, Structure and DFT calculation of chlorimuron-ethyl. Asian J. Chem. 2012, 24, 2808-2810.

6. Liu, X.H.; Weng, J.Q.; Tan, C.X. Synthesis, crystal structure, and fungicidal activity of 5-(4-cyclopropyl-5-((3-fluorobenzyl)thio)-4H-1,2,4-triazol-3-yl)-4-methyl-1,2,3-thiadiazole. J. Chem. 2013, 2013, doi:10.1155/2013/306361.

7. Tong, J.Y.; Shi, Y.X.; Liu, X.H.; Sun, N.B.; Li, B.J. Synthesis and fungicidal activity of 1,2,4-triazole derivatives containing 2-fluorophenyl moiety. Chin. J. Org. Chem. 2012, 32, 2373-2377.

8. Weng, J.Q.; Wang, L.; Liu, X.H. Synthesis, Crystal structure and herbicidal activity of a 1,2,4-triazol-5(4H)-one derivative. J. Chem. Soc. Pakistan 2012, 34, 1248-1252.

9. Liu, X.H.; Zhao, W.G.; Wang, B.L.; Li, Z.M. Synthesis, Bioactivity and DFT structure-activity relationship study of novel 1,2,3-thiadiazole derivatives. Res. Chem. Intermed. 2012, 38, 1999-2008.

10. Sun, N.B.; Tong, J.Y.; Wu, H.K. Synthesis and fungicidal activity of 1,3,4-oxadiazole derivatives containing pyrazole moiety Chin. J. Org. Chem. 2013, 33, 101-105.

11. Tan, C.X.; Shi, Y.X.; Weng, J.Q.; Liu, X.H.; Li, B.J.; Zhao, W.G. Synthesis and antifungal activity of 1,2,4-triazole derivatives containing cyclopropane moiety. Lett. Drug Des. Discov. 2012, 9, 431-435.

12. El-Serwy, W.S.; Mohamed, N.A.; Abbas, E.M.; Abdel-Rahman, R.F. Synthesis and anti-inflammatory properties of novel 1,2,4-triazole derivatives. Res. Chem. Intermed. 2013, 39, 2543-2554.

13. Kocyigit-Kaymakcioglu, B.; Celen, A.O.; Tabanca, N.; Ali, A.; Khan, S.I.; Khan, I.A.; Wedge, D.E. Synthesis and biological activity of substituted urea and thiourea derivatives containing 1,2,4-triazole moieties. Molecules 2013, 18, 3562-3576.

14. Liu, X.H.; Tan, C.X.; Weng, J.Q. Synthessis, Dimeric crystal structure and fungicidal activity of 1-(4-methylphenyl)-2-(5-((3,5-dimethyl-1h-pyrazol-1-yl)methyl)-4-phenyl-4h-1,2,4-triazol-3ylthio)ethanone. Phosphorus, Sulfur Silicon Relat. Elem. 2011, 186, 558-564.

15. Tong, J.Y.; Wu, H.K.; Sun, N.B.; Liu, X.H. Synthesis, Crystal structure and biological activity of a new 1,2,4-triazole derivative. Chin. J. Struct. Chem. 2013, 32, 607-611. 
16. Ke, W.; Sun, N.B.; Wu, H.K. Microwave assistant synthesis, crystal structure and biological activity of a 1,2,4-triazole compound. J. Chem. Soc. Pakistan 2013, 35, 1239-1244.

17. Al-Omar, M.A.; Al-Abdullah, E.S.; Shehata, I.A.; Habib, E.E.; Ibrahim, T.M.; El-Emam, A.A. Synthesis, antimicrobial, and anti-inflammatory activities of novel 5-(1-adamantyl)-4arylideneamino-3-mercapto-1,2,4-triazoles and related derivatives. Molecules 2010, 15, 2526-2550.

18. Franklim, T.N.; Freire-de-Lima, L.; Diniz, J.D.S.; Previato, J.O.; Castro, R.N.; Mendonca-Previato, L.; de Lima, M.E.F. Design, Synthesis and trypanocidal evaluation of novel 1,2,4-triazoles-3-thiones derived from natural piperine. Molecules 2013, 18, 6366-6382.

19. Benci, K.; Mandic, L.; Suhina, T.; Sedic, M.; Klobucar, M.; Pavelic, S.K.; Pavelic, K.; Wittine, K.; Mintas, M. Novel coumarin derivatives containing 1,2,4-triazole, 4,5-dicyanoimidazole and purine moieties: Synthesis and evaluation of their cytostatic activity. Molecules 2012, 17, 11010-11025.

20. Yamada, K.; Yoshizawa, Y.; Oh, K. Synthesis of 2RS,4RS-1-[2-phenyl-4-[2-(2-trifluromethoxyphenoxy)-ethyl]-1,3-dioxolan-2-yl-methyl]-1H-1,2,4-triazole derivatives as potent inhibitors of brassinosteroid biosynthesis. Molecules 2012, 17, 4460-4473.

21. Liu, X.H.; Weng, J.Q.; Tan, C.X. Microwave synthesis and biological activity of hydrazone derivatives containing 1,2,3-thiadiazole. Asian J. Chem. 2011, 23, 4064-4066.

22. Tan, C.X.; Weng, J.Q.; Liu, Z.X.; Liu, X.H.; Zhao, W.G. Synthesis, crystal structure, and fungicidal activity of a novel 1,2,3-thiadiazole compound. Phosphorus, Sulfur Silicon Relat. Elem. 2012, 187, 990-996.

23. Dong, W.L.; Liu, Z.X.; Liu, X.H.; Li, Z.M.; Zhao, W.G. Synthesis and antiviral activity of new acrylamide derivatives containing 1,2,3-thiadiazole as inhibitors of hepatitis B virus replication, Eur. J. Med. Chem. 2010, 45, 1919-1926.

24. Ke, W.; Sun, N.B.; Wu, H.K. Synthesis, Crystal structure and biological activity of a novel 1,2,3-thidiazole compound. J. Chem. Soc. Pakistan 2013, 35, 1233-1238.

25. Liu, X.H.; Pan, L.; Weng, J.Q.; Tan, C.X.; Li, Y.H.; Wang, B.L.; Li, Z.M. Synthesis, structure, and biological activity of novel (oxdi/tri)azoles derivatives containing 1,2,3-thiadiazole or methyl moiety. Mol. Divers. 2012, 16, 251-260.

26. Hayat, F.; Salahuddin, A.; Zargan, J.; Azam, A. Synthesis, characterization, antiamoebic activity and cytotoxicity of novel 2-(quinolin-8-yloxy) acetohydrazones and their cyclized products (1,2,3thiadiazole and 1,2,3-selenadiazole derivatives). Eur. J. Med. Chem. 2010, 45, 6127-6134.

27. Padmavathi, V.; Mahesh, K.; Nagendra Mohan, A.V.; Padmaja, A. Synthesis and bioassay of oxazolyl/thiazolyl selenadiazoles, thiadiazoles and diazaphospholes. Chem. Pharm. Bull. 2009, 57 561-566.

28. Sun, N.B.; Liu, X.H.; Weng, J.Q.; Tan, C.X. An unexpected product n-(3-((2-fluorobenzyl)thio)5-methyl-4h-1,2,4-triazol-4-yl)acetimidamide: Synthesis and structure analysis. J. Chem. Soc. Pakistan 2013, 35, 499-502.

29. Tan, C.X.; Shi, Y.X.; Weng, J.Q.; Liu, X.H.; Zhao, W.G.; Li, B.J. Synthesis and antifungal activity of novel 1,2,4-triazole derivatives containing 1,2,3-thiadiazole moiety. J. Heterocycl. Chem. 2013, doi:10.1002/jhet.1656.

30. Liu, X.H.; Weng, J.Q.; Tan, C.X.; Pan, L.; Wang, B.L.; Li, Z.M. Synthesis, biological activities and dft calculation of alpha-aminophosphonate containing cyclopropane moiety. Asian J. Chem. 2011, 23, 4031-4036. 
31. Liu, X.H.; Chen, P.Q.; Wang, B.L.; Li, Y.H.; Wang, S.H.; Li, Z.M. Synthesis, bioactivity, theoretical and molecular docking study of 1-cyano-N-substituted-cyclopropanecarboxamide as ketol-acid reductoisomerase inhibitor. Bioorg. Med. Chem. Lett. 2007, 17, 3784-3788.

32. Frisch, M.-J.; Trucks, G.-W.; Schlegel, H.-B.; Scuseria, G.-E.; Robb, M.-A.; Cheeseman, J.-R.; Montgomery, J.-A. Jr.; Vreven, T.; Kudin, K.-N.; Burant, J.-C.; et al. Gaussian 03, Revision C. 01; Gaussian, Inc.: Wallingford, CT, USA, 2004.

Sample Availability: Samples of the compounds are available from the authors.

(C) 2013 by the authors; licensee MDPI, Basel, Switzerland. This article is an open access article distributed under the terms and conditions of the Creative Commons Attribution license (http://creativecommons.org/licenses/by/3.0/). 